\title{
Ex-situ Li plating detection on graphite anodes in extremely fast-charged lithium-ion batteries using simultaneous neutron and $X$-ray tomography
}

Maha Yusuf ${ }^{1}$, Jacob LaManna ${ }^{2}$, Partha Paul ${ }^{3}$, David Agyeman-Budu ${ }^{3}$, Michael Toney ${ }^{4}$ and Johanna Weker 5

${ }^{1}$ Stanford Universityy, Stanford, California, United States, ${ }^{2}$ National Institute of Standards and Technology, Gaithersburg, Maryland, United States, ${ }^{3}$ Stanford Synchrotron Radiation Light source, Menlo Park, California, United States, ${ }^{4}$ University of Colorado Boulder, Boulder, Colorado, United States, ${ }^{5}$ Stanford Synchrotron Radiation Light source, Menlo Park, United States

Problem: The long charging times required for lithium-ion batteries (LIBs) constitute a major bottleneck in the widespread deployment of battery electric vehicles (BEVs). Currently available BEVs cannot charge at rates that offer a similar experience to that of refueling a gasoline car at a gas station. Among the state-of-the-art BEVs, Tesla vehicles take 1-12 hours to charge at the fastest recharge rates of $120 \mathrm{~kW}$ through Supercharger stations. ${ }^{1}$ Therefore, there is a global push to enable extreme fast charging (XFC) that would reduce LIB charging times to 10-15 minutes. ${ }^{2}$ However, existing LIBs cannot achieve this XFC goal without significantly reducing battery performance. One of the identified XFC failure pathways is a phenomenon known as "lithium (Li) plating," which severely limits battery capacity over lifetime and eventually leads to battery failure. ${ }^{3}$ Thus, understanding the origin and characteristics of Li plating on graphite anodes is crucial to developing XFC batteries.

Approach: In this work, we used simultaneous neutrons and X-ray-based tomography (NeXT) 4 as a non-destructive imaging modality to visualize Li plating across three dimensions on graphite anode ex-situ after XFC in LIBs. Since X-rays are sensitive to the electron density and neutrons to the nuclear density of the material, NeXT readily separates battery anode components such as Li, graphite, and copper, due to the complementary interaction of the two imaging probes with matter. Higher-energy X-rays are needed to penetrate the metallic components in a battery such as the copper current collector. However, X-rays lack the sufficient imaging contrast to differentiate low-Z elements, especially at high energies. Here, neutrons provide the sensitivity to differentiate graphite from $\mathrm{Li}$ due to the larger difference in their relative neutron crosssections.

Methodology: We performed proof-of-concept multi-modal imaging experiments at the NeXT system located on the BT-2 imaging beamline at the National Institute of Standards and Technology Center for Neutron Research (NCNR). ${ }^{4}$ We characterized pristine and cycled graphite anode strips containing plated Li. For cycled anode strips, we disassembled the battery pouch cells and harvested graphite anodes at fully discharged condition after these were cycled under XFC conditions, specifically 9 C-rate for 450 cycles. ${ }^{5}$ Our spatial resolution was $\sim 15-20$ $\mu \mathrm{m}$, which was sufficient to pinpoint the location of Li plating within the thickness $(80-100 \mu \mathrm{m})$ of the graphite anode.

Data analysis/Discussion: For data analysis, we first denoised the neutron and X-ray images. Then, we used Livermore Tomography Toolbox, ${ }^{6}$ a fast and user-friendly tomographic reconstruction package developed in LabView, for 3D iterative cone beam reconstruction. Next, we used bivariate histogram phase segmentation ${ }^{7}$ on the reconstructed neutron and $\mathrm{X}$-ray 
images to distinguish graphite/Li and copper from each other (Figure 1). The 2D bivariate histogram used information from both the neutron and X-ray images to differentiate different battery materials. Since we imaged the graphite strip from top to bottom, we correlated the growth of Li plating evident in certain segmented colorized slices to the spatial location on the optical image of the cycled anode (Figure $2(A)$ ). In addition, we stitched the 2D slices to create $3 \mathrm{D}$ visualizations that show depth of Li plating on cycled anode (Figure 2(B)). Here, we note few caveats: 1) during the sample preparation, Li was exposed to air and formed $\mathrm{LiOH}$. Therefore, in this work, Li corresponds to "LiOH" plating; 2) owing to the ex-situ nature of the sample, copper delaminated itself from the graphite anode (visible in 2D slices); and 3) flat sample geometry made it particularly challenging to segment graphite from $\mathrm{LiOH}$ during image processing.

Conclusions/Future work: Our ex-situ work demonstrated the feasibility of NeXT as an advanced characterization method for studying Li plating in XFC batteries. However, to answer when and why does Li plating happen, we are working on in-situ NeXT imaging of full LIB coin cells during XFC. To simplify our experimental and data analysis approach, we are designing a neutron-imaging-friendly LIB coin cell. Specifically, we will investigate the effect of cycling rate on the extent and heterogeneity of Li plating as a function of time, and thickness of graphite anode. We believe that addressing this fundamental question will help inform improved battery designs that may reduce Li plating during XFC in LIBs. Ultimately, these improvements based on our research findings may help charge an BEV similar to filling up at a gas station.

Author Contributions: M.Y., J.M.L., P.P., and J.W., performed the NeXT imaging experiments. M.Y., and J.M.L., conducted image processing and data analysis. M.Y., J.M.L., P.P., D.N.A., M.F.T., and J.W., discussed the experimental results and reviewed the paper. The paper was written through contributions of all authors. All authors have given approval to the final version of the paper.

Acknowledgements: Funding was provided from the Vehicle Technologies Office of the U.S. Department of Energy's Office of Energy Efficiency and Renewable Energy under the guidance of the Advanced Battery Cell Research Program (eXtreme fast charge Cell Evaluation of Lithiumion batteries, XCEL). Experiments were performed at the BT-2 imaging beamline at NCNR. Authors are grateful to Andrew N. Jansen and Alison R. Dunlop at the Cell Analysis, Modeling, and Prototyping Facility at Argonne National Laboratory for designing, assembling, and forming the LIB pouch cells. We would also like to acknowledge Tanvir R. Tanim and Eric J. Dufek at the Idaho National Laboratory for carrying out electrochemical cycling of LIB pouch cells. M.Y. is thankful to the Schlumberger Faculty for the Future, and DARE (Diversifying Academia, Recruiting Excellence) doctoral fellowships.

Disclaimers:

The authors declare no competing financial interest. 

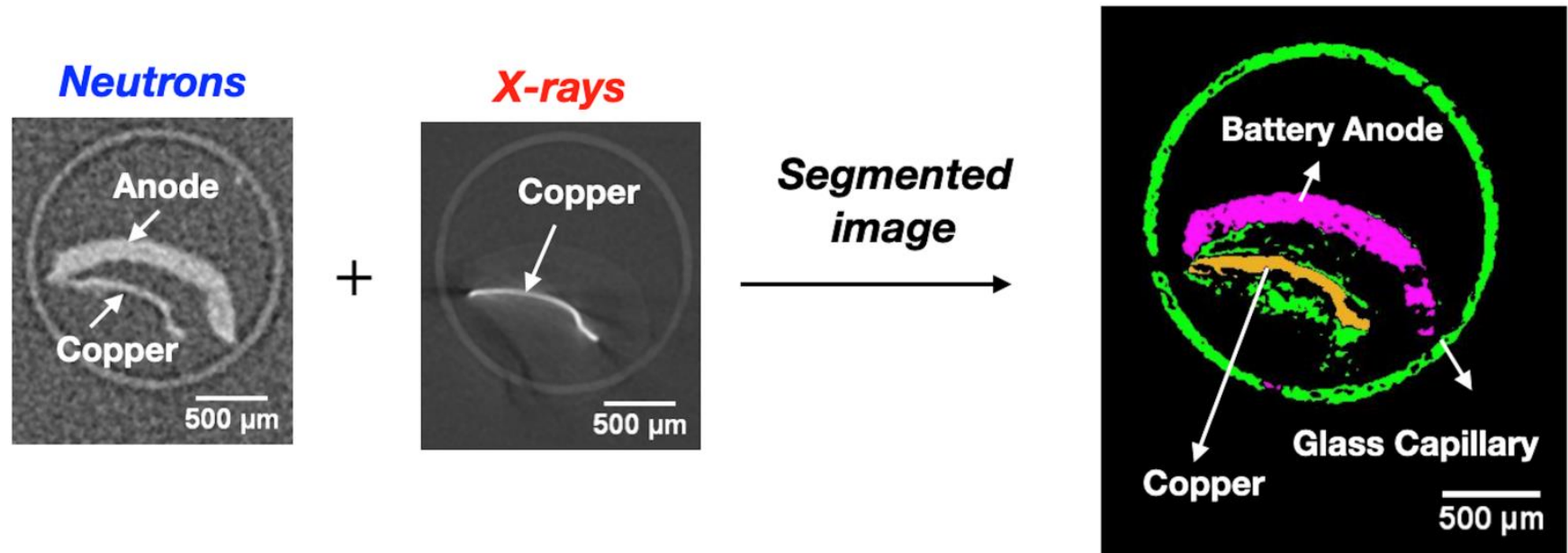

Figure 1. 2D grayscale image (neutrons and $x$-rays) of a cycled graphite anode showing different battery components. Bivariate histogram phase segmentation was used to convert grayscale images to segmented colorized image as shown on the right-hand side.

(A) 2D segmented colorized slices of a 3D volume
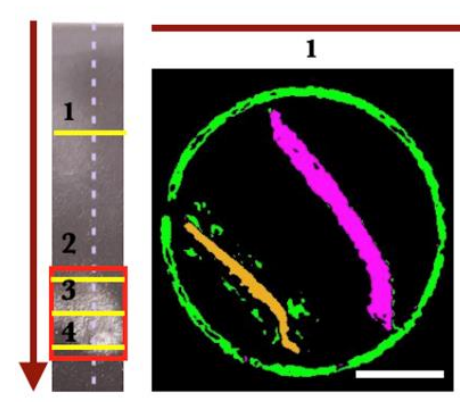
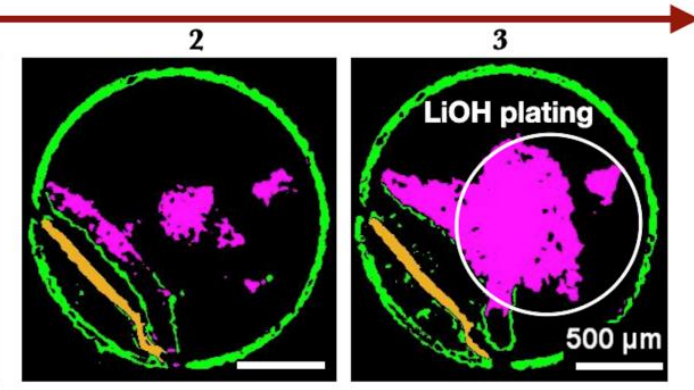

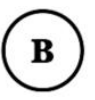

3D visualization shows
depth of $\mathrm{LiOH}$ plating

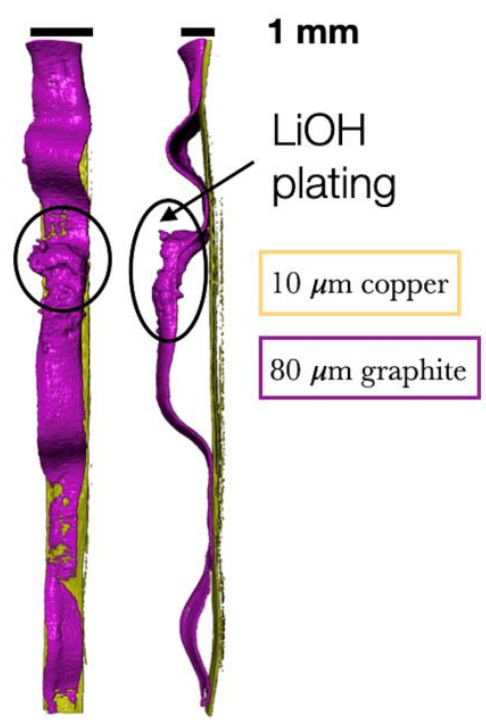

Figure 2. Figure 2. (A) Optical image of a cycled graphite strip showing Li deposition inside a red highlighted box (right-hand side). 2D segmented colorized images of a 3D volume can be correlated to the spatial location on the optical image of the cycled anode. For example, Slice 3 showing growth of $\mathrm{LiOH}$ plating corresponds to the yellow line labelled 3 on the graphite strip. (B) 3D visualizations of the cycled graphite anode show depth of LiOH plating.

\section{References:}

1. Ahmed, Shabbir, et al. "Enabling fast charging-A battery technology gap assessment." Journal of Power Sources 367 (2017): 250-262.

2. Liu, Yayuan, Yangying Zhu, and Yi Cui. "Challenges and opportunities towards fast-charging battery materials." Nature Energy 4.7 (2019): 540-550. 
3. Tomaszewska, Anna, et al. "Lithium-ion battery fast charging: A review." ETransportation 1 (2019): 100011.

4. LaManna, Jacob M., et al. "Neutron and X-ray Tomography (NeXT) system for simultaneous, dual modality tomography." Review of Scientific Instruments 88.11 (2017): 113702.

5. Tanim, Tanvir R., et al. "Extreme fast charge challenges for lithium-ion battery: variability and positive electrode issues." Journal of The Electrochemical Society 166.10 (2019): A1926.

6. K. M. Champley, "Livermore Tomography Tools (LTT) technical manual," LLNL, Livermore, CA, USA, Tech. Rep. LLNL-SM-687016, Dec. 2016.

7. LaManna, J. M., et al. "NIST NeXT: a system for truly simultaneous neutron and X-ray tomography." Hard X-Ray, Gamma-Ray, and Neutron Detector Physics XXII. Vol. 11494. International Society for Optics and Photonics, 2020. 